\title{
Origin of the Wang-Sheeley-Arge solar wind model
}

\author{
Neil R. Sheeley Jr. \\ Naval Research Laboratory, Washington DC 20375-5352, USA \\ Correspondence to: Neil R. Sheeley Jr. (solsheeley@verizon.net)
}

Received: 28 November 2016 - Revised: 22 February 2017 - Accepted: 15 March 2017 - Published: 31 March 2017

\begin{abstract}
A correlation between solar wind speed at Earth and the amount of magnetic field line expansion in the corona was verified in 1989 using 22 years of solar and interplanetary observations. We trace the evolution of this relationship from its birth 15 years earlier in the Skylab era to its current use as a space weather forecasting technique. This paper is the transcript of an invited talk at the joint session of the Historical Astronomy Division and the Solar Physics Division of the American Astronomical Society during its 224th meeting in Boston, MA, on 3 June 2014.
\end{abstract}

\section{Introduction}

This is the 25th anniversary of the origin of the WangSheeley-Arge (WSA) model. It occurred in 1989 when there was no internet as we now know it, no cell phones, and no Google, and Apple stock was selling for $\$ 45$ per share. Alphonse Stirling reminded me that it was also the year of the World Series earthquake in San Francisco. And the Berlin Wall came down on 9 November.

What is the WSA model? It is a procedure for deriving solar wind speed from maps of the photospheric magnetic field. It is based on the idea that, far from the Sun, the speed of the solar wind depends on the path that the wind took as it passed through the lower corona, as illustrated in Fig. 1. If the flux tube that guided the flow flares out, then the distant speed is slow. But if the flux tube remains focused, then the distant speed is fast. The amount of expansion is obtained from a current-free extension of the photospheric field.

\section{Four milestones}

This road through 1989 had four principal milestones. The first milestone occurred on 1 June (25 years prior to the start of this meeting) when I was defending the Office of Naval Research (ONR) Contract Research Program in Solar Physics before an external review committee. I linked the ONR work to our own Naval Research Laboratory (NRL) research by saying that we used the contractor magnetograms to derive the locations of open field regions and thus coronal holes. And we used our new flux-transport program, created

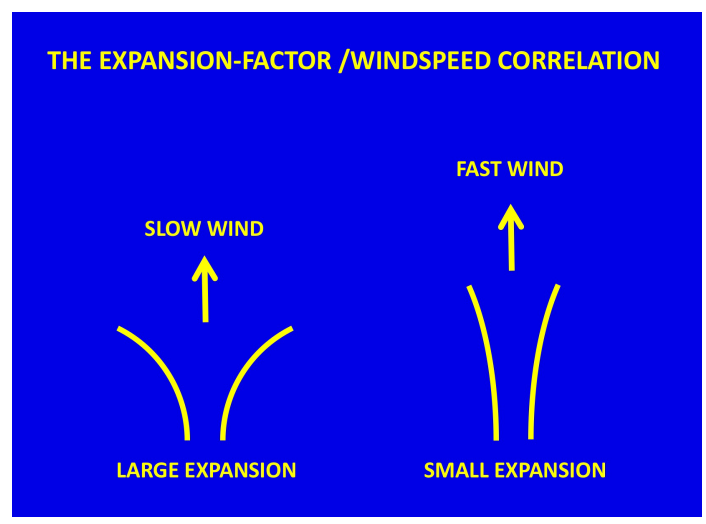

Figure 1. A cartoon, illustrating that fast wind comes from magnetic flux tubes with small coronal expansion and that slow wind comes from flux tubes with large coronal expansion.

at NRL by Jay Boris and Rick DeVore, to evolve the magnetic maps forward in time by 1 or 2 months so that we could calculate the locations of future coronal holes. Because coronal holes were sources of high-speed solar wind streams and associated geomagnetic disturbances, I argued that this procedure ought to be useful for forecasting geomagnetic activity.

The committee liked the leveraging to the NRL program, but it wanted the procedure to be available at the US Air Force (USAF) and National Oceanic and Atmospheric Administration (NOAA) forecasting centers. Consequently, an action item was created, asking me to devise a plan to make 


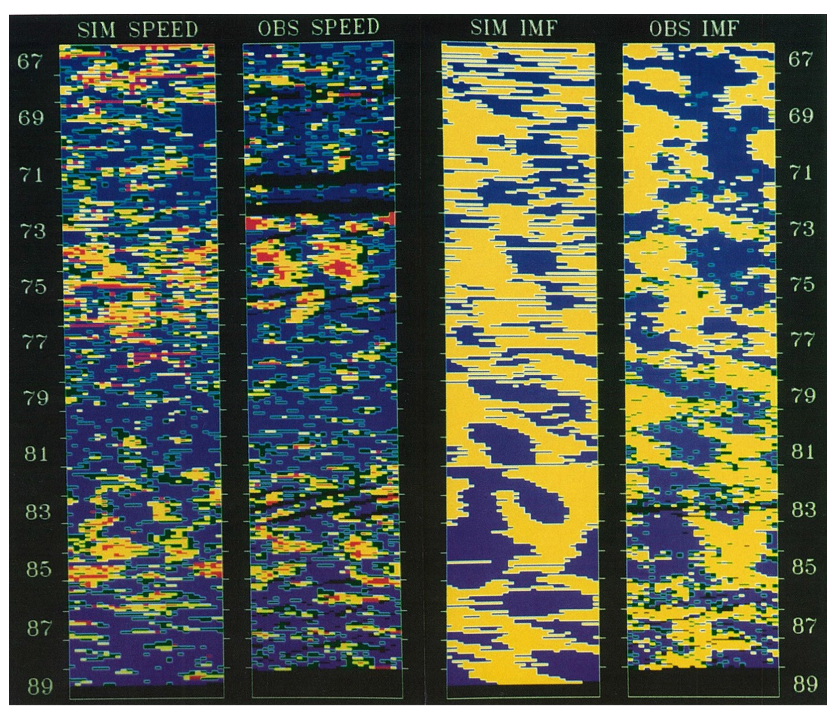

Figure 2. The original Bartels comparison of simulated and observed wind speed (left columns) and interplanetary magnetic field (right columns) from the paper by Wang and Sheeley (1990).

our coronal-hole forecasting procedure operational at those centers.

The second, even more important milestone occurred during August as Yi-Ming Wang was trying to find a magneticfield quantity that was correlated with the solar wind speed. Previously, we had some success comparing coronal holes and wind speed in Bartels displays (Sheeley et al., 1976), but Yi-Ming was obtaining poor correlations with most quantities like the strength of the photospheric field or sourcesurface field at the sub-Earth point, or the angular distance between this sub-Earth point and the magnetic neutral line.

The situation changed when Yi-Ming read an old paper by Levine et al. (1977) in the Journal of Geophysical Research (JGR). Levine et al. (1977) had used magnetic field measurements obtained at Kitt Peak to make current-free calculations of the coronal magnetic field during the 9-month Skylab mission and compared the resulting coronal magnetic field topology with in situ measurements of solar wind speed. They found that the fastest streams were correlated with flux tubes that expanded least during their passage through the lower corona, and concluded that the divergence of the field plays "an apparently crucial role" in the acceleration of the solar wind. When Yi-Ming compared the coronal expansion factor with the observed solar wind speed during the 22-year interval 1967-1989, he obtained the result shown in the left panels of Fig. 2. The correlation extended throughout the sunspot cycle and did not require separate treatments of data at sunspot maximum and minimum.

The Levine et al. (1977) result was largely ignored at the time it was published. It was a product of the Skylab Workshop on Coronal Holes where Munro and Jackson (1977) emphasized that the polar coronal holes expand by an enormous

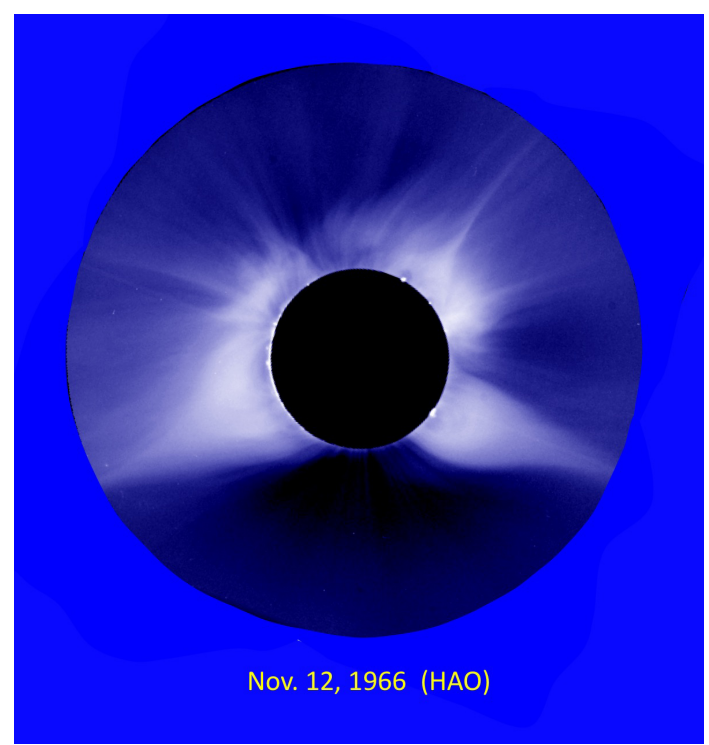

Figure 3. High Altitude Observatory image of the 12 November 1966 solar eclipse. Munro and Jackson (1977) used this image to deduce that the solid angle of the magnetic field in the south polar coronal hole increased by a factor of 7 between 1 and 3 solar radii.

factor of 7 in solid angle, as seen at the South Pole in Fig. 3. Thus, many researchers associated large expansion with the fast wind that was known to come from coronal holes. Also, in 1989, solar wind theory provided little support for an inverse relation between the coronal expansion factor and the speed of the wind. Thermally driven winds showed only a weak dependence on the expansion factor (Leer et al., 1982) and some Alfven wave models predicted that a high coronal expansion factor would produce a faster rather than a slower wind speed (Esser et al., 1986).

As Levine et al. knew and Yi-Ming recognized, the important quantity was not the expansion of the entire coronal hole, but the expansion of the individual flux tubes within the hole. As shown in Fig. 4, the individual flux tubes of an axisymmetric dipole field have relatively small expansions, except toward the edge of the hole where the tubes flare out considerably. Because this equatorial streamer-belt/currentsheet region was known to be associated with slow wind, it followed that the solar wind speed was inversely related to the amount of expansion that occurs in individual flux tubes of the coronal hole. However, the question of how much slow wind comes from rapidly expanding flux tubes at the edge of a coronal hole and how much comes from the tops of the stretched coronal loops in the streamer belt remains an active subject of study (Antiochos et al., 2011; Wang, 2012; Abbo et al., 2016).

Yi-Ming's discovery was the break that we needed. When I told my ONR contact, Ken Davis, about the new result, he asked me to brief Bruce Robinson, the ONR Director of Science, in a meeting on 24 August. As you can see from my 


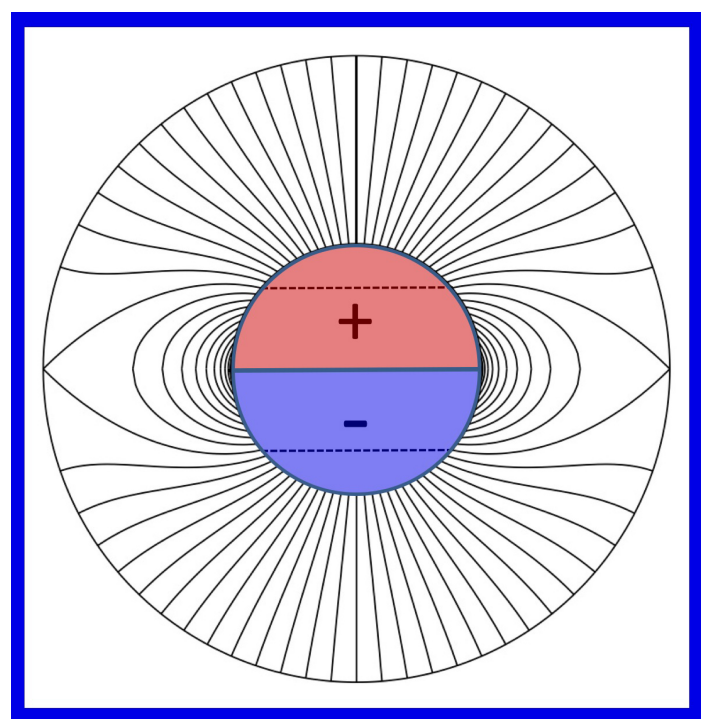

Figure 4. Current-free field lines of a photospheric dipole field, constrained to be radial at 2.5 solar radii to simulate the stretching effect of the coronal expansion. These field lines show the increased flux-tube expansion toward the edge of the polar coronal holes.

briefing notes in Fig. 5, the words "new result last week" pinpoint the discovery to a date around 17 August 1989. In addition, the notes contain three points that summarized our status at that time: "seem to have found a way to derive wind speed from magnetograms", "a missing link in our forecasting capability", and "relevant to the request to make our model operational". This briefing led to the third milestone 4 days later on 28 August 1989, which was to give ONR the requested plan to make our forecasting model operational at the USAF and NOAA forecasting centers. The expansion factor-wind speed relation was a central part of that plan.

The fourth milestone was to publish the result and make it familiar to the scientific community. As shown in Fig. 6, our paper, Wang and Sheeley (1990), was received at the Astrophysical Journal on 5 October 1989. The paper was accepted on 5 December, and the next day I described our result in Session SM31B of the American Geophysical Union (AGU) fall meeting in San Francisco. I can still recall discussing implications of the result with Ernie Hildner in the hallway afterwards. The paper appeared in print on 1 June 1990, exactly 1 year after the external review at ONR.

During the next year, we pursued the implications of the inverse correlation between wind speed and coronal expansion factor (Sheeley and Wang, 1991) and tried to understand why there should be such a correlation, which was not supported by theoretical analyses at that time (Leer et al., 1982). Yi-Ming recognized that a uniform distribution of mechanical energy flux at the base of a coronal hole would produce a non-uniform coronal distribution with the larger values of energy flux on lower-expansion flux tubes. In contrast, the mass flux is distributed nearly uniformly in the corona be-

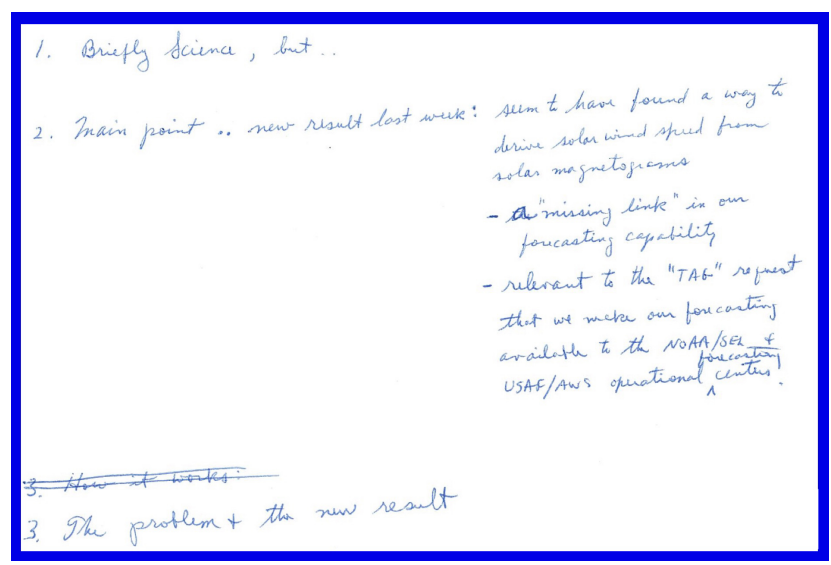

Figure 5. Notes prepared for my 24 August 1989 meeting with the ONR Science Director. They pinpoint Yi-Ming's discovery around 17 August 1989.

cause it is determined from a balance between gravity and thermal pressure. Consequently, the ratios of mechanical energy flux to mass flux are enhanced on low-expansion flux tubes, which therefore have correspondingly larger values of asymptotic solar wind speed.

We published this result on 1 May 1991 (Wang and Sheeley, 1991), and then discussed the idea during 16-20 September 1991 at the Seventh Solar Wind Conference in Goslar, Germany (Wang and Sheeley, 1991; Sheeley, 1992). Figure 7 shows us with three of our solar colleagues (Ken Dere, Ana Nash, and Ken Widing) in Goslar at that time. Ana made the original Bartels display shown in Fig. 2.

\subsection{Transition to use}

The original plan of making our forecasting procedure operational was eventually carried out. During 1993-1995, we sent monthly updates of our calculations to Capt. Paul Gehred at the USAF/AWS in Boulder, CO, who compared geomagnetic forecasts based on those calculations with the recurrencebased forecasts that were being used at the operational centers at that time. I lost contact with Paul in mid-November 1995, when he moved to the Air Force Research Lab in Dayton, $\mathrm{OH}$, but his reports indicated that he had obtained slightly better results with the model-based forecasts than with the recurrence-based ones.

The process entered a new phase around the time of the AGU meeting in December 1995, probably in an encounter between Bob McCoy (who had been managing the ONR program since I left it in 1994), Ernie Hildner (the NOAA/Space Environment Laboratory (SEL) Director), and Todd Hoeksema (a member of the Wilcox Solar Observatory (WSO) contractor team). I think that Bob came up with the idea to reduce the funding of each contractor team by a little bit and use the savings to fund a post doc at NOAA/SEL to make the Wang-Sheeley (WS) model operational. It was really a bril- 
THE ASTROPHYSICAL JOURNAL 355, 726-732, 1990 June1

SOLAR WIND SPEED AND CORONAL FLUX-TUBE EXPANSION

Y.-M. WANG AND N. R. SHEELEY, JR.

E. O. Hulburt Center for Space Research, Naval Research Laboratory

Received 1989 October 5 ; accepted 1989 December 5

\section{ABSTRACT}

The hypothesis that the solar wind speed at $1 \mathrm{AU}$ and the rate of magnetic flux-tube expansion in the corona are inversely correlated is shown to be consistent with observations extending over the last 22 years This empirical relationship allows the daily wind speeds at Earth to be predicted from a current-free extrapolation of the observed photospheric field into the corona. We attribute the narrow boundaries of high-speed wind streams to steep gradients in the flux-tube expansion rates at the edges of coronal holes. When a heliospheric current sheet is included in the model, we find that the flux tubes near the hole axis, although diverging more slowly than those near the hole boundary in the corona, have undergone the greatest net expansion at $1 \mathrm{AU}$, an effect consistent with the low densities within high-speed streams.

Subject headings: Sun: corona - Sun: solar wind

Figure 6. The title page of our paper relating solar wind speed to the coronal expansion factor as published in the 1 June 1990 issue of The Astrophysical Journal.

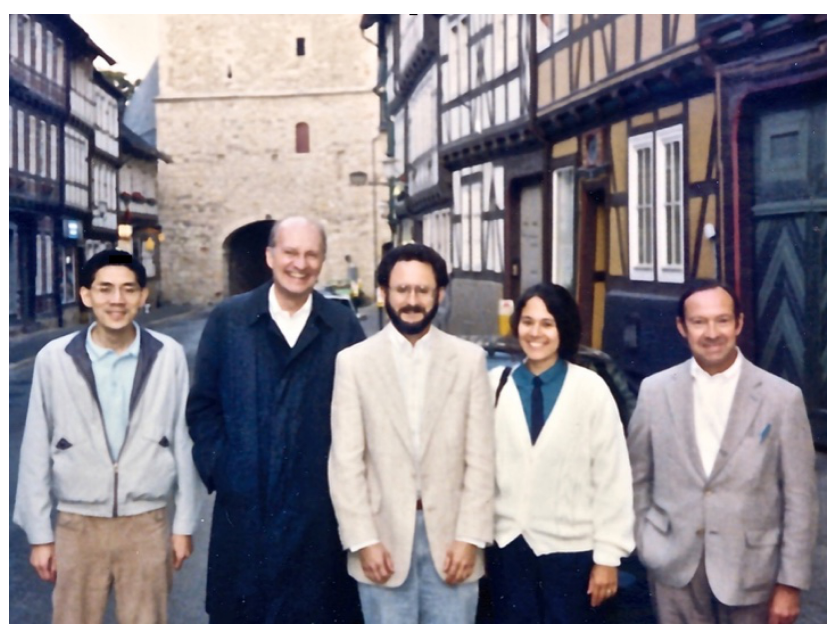

Figure 7. September 1991 photo, showing (left to right) Yi-Ming Wang, the author, Ken Dere, Ana Nash, and Ken Widing in Goslar, Germany, during Solar Wind Seven. The photo was taken by Don Michels, who was coordinating international efforts for the Large Angle Spectrometric Coronagraph (LASCO). LASCO was launched as part of the Solar and Heliospheric Observatory (SOHO) 4 years later on 2 December 1995, and is still operating successfully.

liant idea. The plan would not only provide additional work on the model, but it would also save the dwindling ONR solar program for several more years. For Ernie, it would bring additional funding to NOAA/SEL, and for Todd and the other contractors, it would mean the continuation of most of their ONR support. So when NOAA/SEL scientist Vic Pizzo faxed me a copy of a 30 January 1996 NOAA proposal to fund a post doc for upgrading the Wang-Sheeley solar wind prediction model, I strongly recommended it to Bob McCoy.

Nick Arge became the new post doc and joined NOAA/SEL in early October 1996. When he visited NRL in late November, we taught him to use our codes and gave him the software to take back to Boulder. Sometime in 1997, he created the WS web site with the ONR and NOAA logos shown in Fig. 8. During 1997-2003, Nick continued to make essential contributions to the development, implementation, and improvement of the model (Arge and Pizzo, 2000; Arge et al., 2004), which resulted in the name WSA.

In 2004, Nick suggested that the coronal portion of the WSA model be coupled with the 3-D MHD solar wind model that Dusan Odstrcil had developed for calculating the outward evolution of the solar wind. The combined effort could be done as part of the Center for Integrated Space Weather Modeling (CISM) effort led by Boston University and supported by the National Science Foundation's Science and Technology Center. The idea was to use the WSA model to derive the wind speed and magnetic field in the vicinity of 20-30 solar radii, where the solar wind is both super-sonic and super-Alfvenic, and then to use this information as the inner boundary condition for the MHD model. Dusan and the CISM leadership team endorsed Nick's idea and supported the effort to combine the two models and develop them further. The MHD model was called ENLIL after the Sumerian god of winds and storms (from EN meaning "lord" and LIL meaning "wind"), so the combined model became known as the WSA-ENLIL model.

Finally, in 2011, Vic Pizzo and his colleagues succeeded in making the WSA-ENLIL model operational at the NOAA/SEL, by that time called the NOAA Space Weather Prediction Center (NOAA/SWPC). This transition to operations is described in a paper in Space Weather (Pizzo et al., 2011), whose title page is shown in Fig. 9. As Pizzo et al. (2011) noted, this transition drew upon contributions from several agencies and institutions, including the Center for Integrated Space Weather Modeling (CISM), the Air Force Research Laboratory (AFRL), the NOAA Space Weather Prediction Center (NOAA/SWPC), the Naval Research Laboratory (NRL), the National Center for Atmospheric Research 


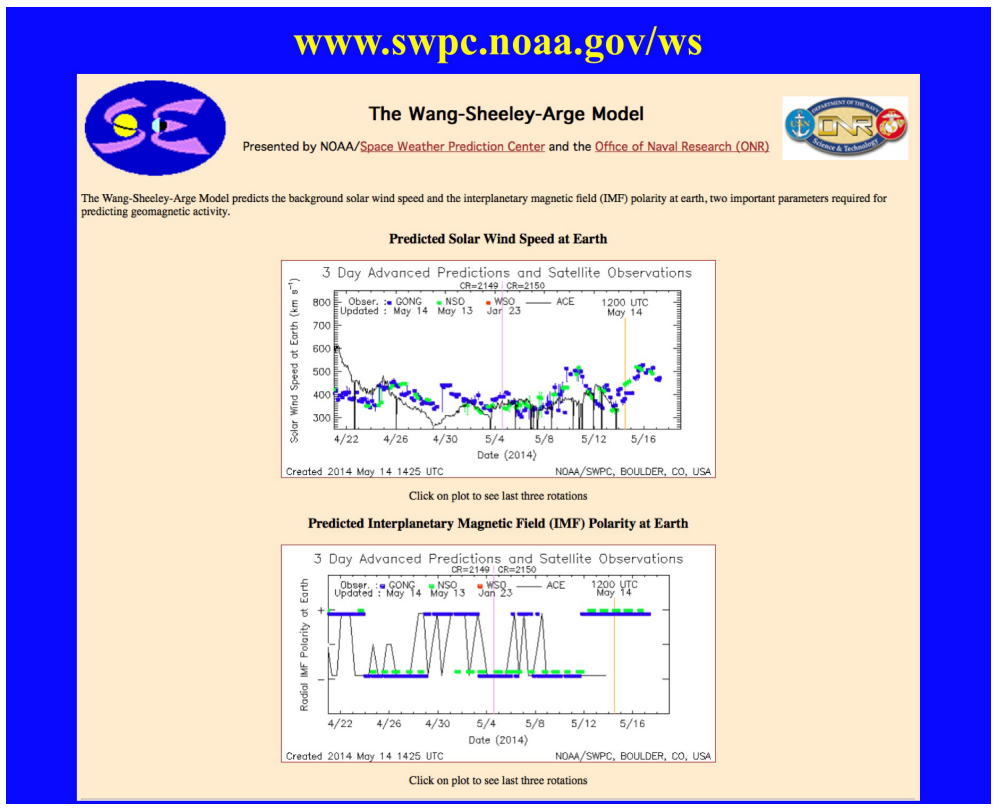

Figure 8. The web site created by Nick Arge, showing a running 3-day forecast of solar wind speed and interplanetary magnetic field (IMF) polarity derived from the Wang-Sheeley-Arge model during a Carrington rotation in April-May 2014.

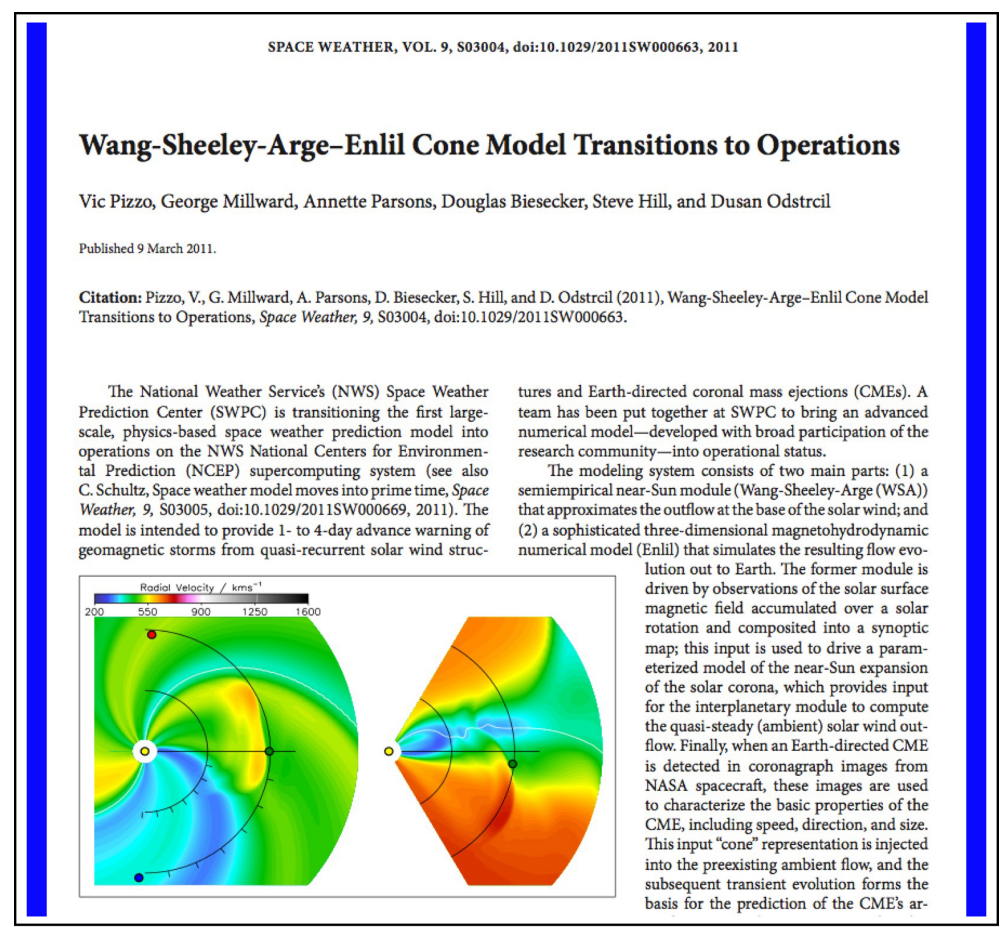

Figure 9. The title page of the announcement by Pizzo et al. (2011) that they had combined the WSA model with the ENLIL MHD model and were now providing forecasts of space weather conditions. 


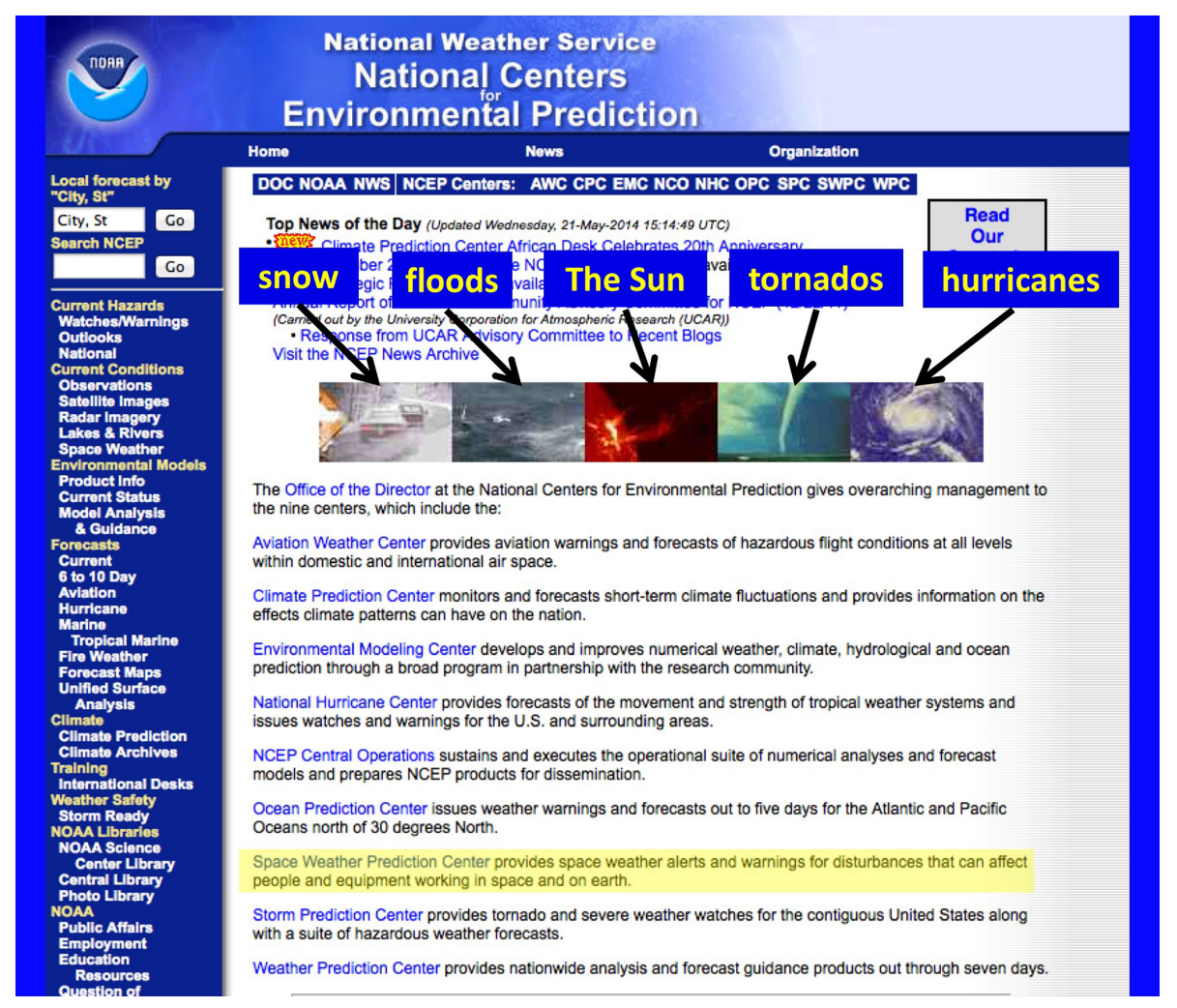

Figure 10. The National Centers for Environmental Prediction web site (http://www.ncep.noaa.gov/) of the National Weather Service, showing solar weather on the same page as snow, floods, tornados, and hurricanes.

(NCAR), the Laboratory for Atmospheric and Space Physics (LASP), the Office of Naval Research (ONR), the Air Force Weather Agency (AFWA), and the Community Coordinated Modeling Center (CCMC).

\subsection{Epilogue}

According to Ernie Hildner, the WSA-ENLIL model is the first operational space weather model. Its output is an official National Weather Service (NWS) product and has all the aspects of other NWS products, including reliability, legal status, and resistance to hacker's corruptions, and it is run on the same NWS computers that run the meteorological models. Nick Arge is proud that the Sun now appears on the same page as snow, floods, tornados, and hurricanes, as you can see on the NWS National Centers for Environmental Prediction (NCEP) web site shown in Fig. 10. Bob McCoy, now Director of the Geophysical Institute in Fairbanks, told me that he regards the WSA-ENLIL model as one of our biggest success stories.

If you click on "Space Weather Prediction Center", you will find a list of "Products and Data". In that list, under "Models", click on "WSA-Enlil Solar Wind Prediction". You will obtain a running 7-day display of predicted plasma density and wind speed at Earth, at the STEREO A and
STEREO B spacecraft, and throughout the inner heliosphere, as shown by the movie in Fig. 11 .

(Harry Warren downloaded the relevant frames from the NWS web site and created the movie used in the talk. Readers of this article can obtain the corresponding movie by going to the NWS web address, http://www.swpc.noaa.gov/ products/wsa-enlil-solar-wind-prediction, and running the model.)

\section{Conclusions}

In summary, during the past 25 years, we have progressed from a position defending long-term synoptic observations of the Sun's magnetic fields to a forecasting position between floods and tornados on the NWS/NCEP web site. But in this glow of success, let us not forget that there were no web sites in 1989 when the model was created, and that the expansion factor-wind speed relation resulted because researchers like Yi-Ming Wang (and Randy Levine and his colleagues before that) were curious about those solar observations. In this audience, I see people who are using similar observations to study the sunspot cycle and solar activity. It is easy to imagine that their research will ultimately lead to a prominent position on a forecasting medium that has not even been invented yet. 


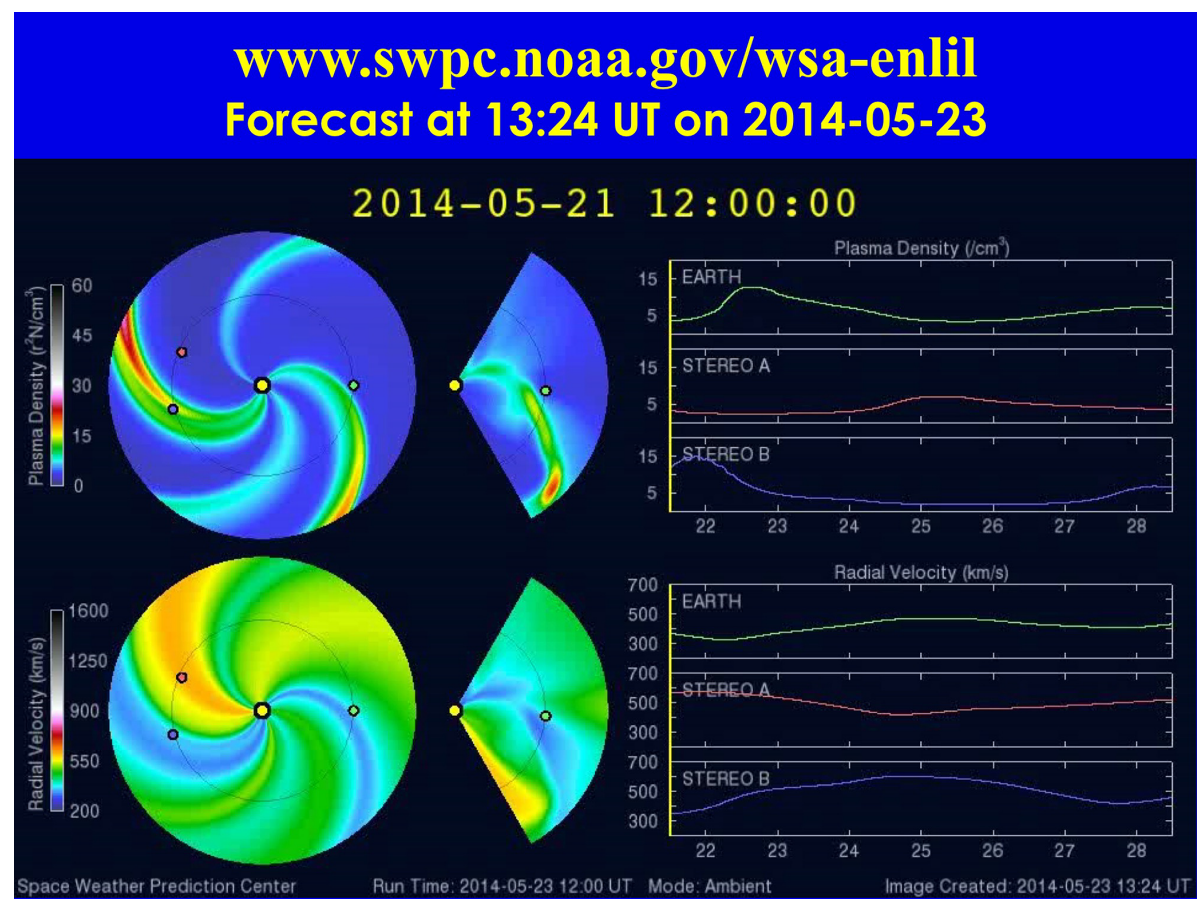

Figure 11. The WSA-ENLIL forecasting model as it appeared on the NOAA/NWS web site in 2014, providing running forecasts of solar wind speed and ion density at Earth and at the STEREO A and B spacecraft. The link now forwards to a new address given by http: //www.swpc.noaa.gov/products/wsa-enlil-solar-wind-prediction.

Code and data availability. WSA-ENLIL movies mentioned in reference to Fig. 11 can be obtained at http://www.swpc.noaa.gov/ products/wsa-enlil-solar-wind-prediction (NOAA, 2017).

The solar magnetic field data used in the creation of Fig. 2 were obtained from the Mount Wilson Observatory and may be found at http://obs.astro.ucla.edu/intro.html (Ulrich, 2017). The interplanetary data were gathered from a variety of separate instruments, but now may be found at https://omniweb.gsfc.nasa.gov/ (NASA, 2017). New versions of the code to combine these data may be found at https://ccmc.gsfc.nasa.gov/ (Chulaki, 2017). Questions concerning the original code should be addressed to Yi-Ming Wang at the address yi.wang@nrl.navy.mil.

Competing interests. The author declares that he has no conflict of interest.

Acknowledgements. I gleaned most of this historical material from my own notes and records, and from my email messages from the 1990s. I am especially grateful to Nick Arge, Bob McCoy, Ernie Hildner, Todd Hoeksema, Vic Pizzo, and Yi-Ming Wang for recent conversations and email messages that helped to fill in some gaps in this historical material. I hope that I have expressed their recollections and opinions accurately. Also, I am grateful to the referees for providing additional help and suggestions. One of the referees, Nick Arge, helped me to describe the formation of the WSA-ENLIL model and the transition to operations during 2004-2011 - activities with which he was directly involved. Financial support was obtained from NASA and CNR.
Edited by: T. Kikuchi

Reviewed by: N. Arge and one anonymous referee

\section{References}

Abbo, L., Ofman, L., Antiochos, S. K., Hansteen, V. H., Harra, L., Ko, Y.-K., Lapenta, G., Li, B., Riley, P., Strachan, L., von Steiger, R., and Wang, Y.-M.: Slow Solar Wind: Observations and Modeling, Space Sci. Rev., 201, 55-108, doi:10.1007/s11214-0160264-1, 2016.

Antiochos, S. K., Mikić, Z., Titov, V. S., Lionello, R., and Linker, J. A.: A Model for the Sources of the Slow Solar Wind, Astrophys. J., 731, 112, doi:10.1088/0004-637X/731/2/112, 2011.

Arge, C. N. and Pizzo, V. J.: Improvement in the prediction of solar wind conditions using near-real time solar magnetic field updates, J. Geophys. Res., 105, 10465-10480, doi:10.1029/1999JA000262, 2000.

Arge, C. N., Luhmann, J. G., Odstrcil, D., Schrijver, C. J., and Li, Y.: Stream structure and coronal sources of the solar wind during the May 12th, 1997 CME, J. Atmos. Sol.-Terr. Phys., 66, 12951309, doi:10.1016/j.jastp.2004.03.018, 2004.

Chulaki, A. (Curator): Community Coordinated Modeling Center, available at: https://ccmc.nasa.gov, last access: 30 March 2017.

Esser, R., Habbal, S. R., Withbroe, G. L., and Leer, E.: A twofluid solar wind model with Alfven waves - Parameter study and application to observations, J. Geophys. Res., 91, 2950-2960, doi:10.1029/JA091iA03p02950, 1986. 
Leer, E., Holzer, T. E., and Fla, T.: Acceleration of the solar wind, Space Sci. Rev., 33, 161-200, doi:10.1007/BF00213253, 1982.

Levine, R. H., Altschuler, M. D., and Harvey, J. W.: Solar sources of the interplanetary magnetic field and solar wind, J. Geophys. Res., 82, 1061-1065, doi:10.1029/JA082i007p01061, 1977.

Munro, R. H. and Jackson, B. V.: Physical properties of a polar coronal hole from 2 to 5 solar radii, Astrophys. J., 213, 874, doi:10.1086/155220, 1977.

NASA Goddard Space Flight Center (Space Physics Data Facility): OmniWeb Plus, https://omniweb.gsfc.nasa.gov/, 30 March 2017.

NOAA Space Weather Prediction Center: WSA-ENLIL Solar Wind Prediction, available at: http://www.swpc.noaa.gov/products/ wsa-enlil-solar-wind-prediction, last access: 30 March 2017.

Pizzo, V., Millward, G., Parsons, A., Biesecker, D., Hill, S., and Odstrcil, D.: Wang-Sheeley-Arge-Enlil Cone Model Transitions to Operations, Space Weather, 9, 03004, doi:10.1029/2011SW000663, 2011.

Sheeley, Jr., N. R.: Coronal holes and solar wind streams during the sunspot cycle, in: Solar Wind Seven, Proceedings of the 3rd COSPAR Colloquium held in Goslar, Germany, 16-20 September 1991, edited by: Marsch, E. and Schwenn, R., 263-271, Pergamon Press (Oxford, New York, Seoul, Tokyo), 1992.

Sheeley Jr., N. R. and Wang, Y.-M.: Magnetic field configurations associated with fast solar wind, Solar Phys., 131, 165-186, doi:10.1007/BF00151752, 1991.
Sheeley Jr., N. R., Harvey, J. W., and Feldman, W. C.: Coronal holes, solar wind streams, and recurrent geomagnetic disturbances - 1973-1976, Solar Phys., 49, 271-278, doi:10.1007/BF00162451, 1976.

Ulrich, R. (UCLA emeritus professor): The 150-Foot Solar Tower at Mt. Wilson Observatory, available at: http://obs.astro.ucla.edu/ intro.html, last access: 30 March 2017.

Wang, Y.-M.: Semiempirical Models of the Slow and Fast Solar Wind, Space Sci. Rev., 172, 123-143, doi:10.1007/s11214-0109733-0, 2012.

Wang, Y.-M. and Sheeley Jr., N. R.: Solar wind speed and coronal flux-tube expansion, Astrophys. J., 355, 726-732, doi:10.1086/168805, 1990.

Wang, Y.-M. and Sheeley Jr., N. R.: Why fast solar wind originates from slowly expanding coronal flux tubes, Astrophys. J. Lett., 372, L45-L48, doi:10.1086/186020, 1991.

Wang, Y.-M. and Sheeley Jr., N. R.: The relationship between solar wind speed and the areal expansion factor, in: Solar Wind Seven, Proceedings of the 3rd COSPAR Colloquium held in Goslar, Germany, 16-20 September 1991, edited by: Marsch, E. and Schwenn, R., 125-128, Pergamon Press (Oxford, New York, Seoul, Tokyo), 1992. 